\title{
A RATE-DISTORTION BASED COMPARISON OF MEDIA-DEPENDENT FEC AND MDC FOR REAL-TIME AUDIO
}

\author{
György Dán, Viktória Fodor and Gunnar Karlsson
}

Department of Signals, Sensors and Systems, KTH, 10044, Stockholm

E-mail: \{gyuri,viktoria,gk\}@s3.kth.se

\begin{abstract}
Applications that require low loss probabilities in today's Internet have to employ some end-to-end errorrecovery mechanism. For interactive applications with strict delay constraints, the delay introduced by the applied schemes has to be low as well. In this paper we compare two schemes proposed for error recovery for real-time audio applications: media-dependent forward error correction (MD-FEC) and multiple description coding (MDC). We conclude that MDC always performs better than MDFEC, and that the average loss probability plays a key role in the choice of the optimal parameters for these schemes. Combining the analytical results with the loss characteristics of measured traces of VoIP calls we conclude that in the current Internet these schemes give considerable gains for streams with a high code rate only, and for these streams MDC can decrease the average distortion significantly better than MD-FEC.
\end{abstract}

\section{INTRODUCTION}

Real-time audio and video communication is becoming more and more important in the Internet. The network quality provided is however undetermined due to unknown packet loss and end-to-end delay distributions. Though much research has been done in recent years, there is no support yet from the network side for QoS in form of resource reservation and call admission control. Thus applications that require low packet loss and delay jitter have to employ some end-to-end mechanisms that compensate for the disturbances introduced by the network. The problem of delay jitter is often solved on the receiver side via adaptive playout algorithms [1,2]. Packet losses can be compensated for on the sender side and the receiver side. On the latter side, receiver-based error concealment algorithms can be used like insertion or interpolation [3, 4]. On the sender side redundant information can be added to the data flow. Whenever a packet is lost, the redundant information can be used to reconstruct the lost information. Forward error correction (FEC) and the recently rediscovered multiple description coding (MDC) have been proposed for this purpose [5, 6].

There are two main directions of FEC design in order to recover from packet losses. One set of solutions use block coding schemes based on algebraic coding, e.g. Reed Solomon coding, and are suitable for high bitrate applications $[7,8,9]$. The other solution, proposed by the IETF and implemented in Internet audio tools like Rat [10] and Freephone [11] is to add a redundant copy of the original packet to one of the subsequent packets. The redundant packet is highly compressed, so that sound quality reconstructed from it is low, but still better than if there were nothing to play out. Proposed ways to improve the performance of the scheme are to increase the time offset between the original packet and the redundant one in order to make them independent with respect to loss [12] and to send multiple redundant copies in subsequent packets [5]. The performance of this media-dependent FEC scheme (MD-FEC) has been evaluated via simulations in [13] and analytically in $[14,15]$. The results show that if the ratio of the traffic implementing MD-FEC is small, streams can benefit from using MD-FEC.

The traditional approach to networking, the separation of source and channel coding, was motivated by Shannon's separation theorem [16, 17]. It states that source coding and channel coding can be performed separately while maintaining optimality. However, Shannon's separation theorem assumes that the available delay is unlimited, which is not true for real-time applications.

MDC addresses the problem of joint source and channel coding. Originally it was designed for the transmission of multiple descriptions of a single source over independent channels. If only one of the descriptions is received, it is used for reconstruction with a certain accuracy. If more than one descriptions are received, then the information from the other descriptions can be used to enhance the accuracy (in contrast to MD-FEC, where the redundant copy can not be used to enhance quality). MDC has been rediscovered recently for use in packet switched networks [6]. Instead of using separate channels, one can time-shift the different descriptions, similarly to the case of MD-FEC. In the general case the amount of information sent over the separate channels (packets) can be different. For singlepath packet networks, which offer identical treatment to all packets, it can be shown that balanced MDC, i.e. the one sending the same amount of information in all packets is optimal [18].

In this paper we compare the potential of MD-FEC and MDC to decrease the average distortion of real-time audio transmitted over error prone networks. We evaluate the 
average distortion analytically for different network conditions and then combine the analytical results with measurements to evaluate the performance of these schemes under realistic network scenarios. We reveal a fundamental relationship between the available source rate, the average loss probability and the optimal ratio of error correction and show that MDC outperforms MD-FEC under all circumstances. Based on the measured loss traces we show that although for low bitrates neither MD-FEC nor MDC can significantly improve the quality over the Internet, for high bitrates they prove to be more efficient, MDC performing considerably better than MD-FEC.

The optimal selection of source and redundancy rate for real-time audio was investigated before in [5] using the Gilbert channel model. The performance of MDC was compared to that of single description coding in the context of content delivery networks via simulations in [19]. The authors considered an average packet loss rate of 5 percent and concluded that MDC can reduce the distortion by up to 20 to 40 percent. The performance of MI-FEC and MDC with 50 percent redundancy was compared in [20] using the Gilbert channel model. As the authors used a fixed redundancy rate for FEC, the comparison gave advantage to MDC by allowing it to adjust to the channel characteristics.

The rest of the paper is organized as follows. In Section 2 we introduce the distortion rate bounds for FEC and MDC. In Section 3 we compare the performance of MD-FEC and MDC in a lossy environment, and in Section 4 we use measured traces to evaluate how efficient these schemes can be under realistic scenarios. We conclude our work in Section 5.

\section{DISTORTION-RATE BOUNDS FOR FEC AND MDC}

When compressing signals, there is always a tradeoff between the size and the accuracy of the representation. This tradeoff, the bounds on achievable rates and distortions, is represented by the distortion-rate and the rate-distortion functions, which depend on the source characteristics and the distortion measure. We consider a memoryless Gaussian source with unit variance and use the mean squared error distortion measure, which is the most common distortion measure. Lately, Gaussian mixture models using the weighted sum of Gaussian densities have found application in speech coding [21, 22]. For a given variance and with respect to the squared error distortion measure, Gaussian sources are the most difficult to compress [23] and thus represent a worst case scenario. The distortionrate function for a Gaussian source with unit variance and squared distortion measure is given as

$$
D(R)=2^{-2 R},
$$

where $R$ is the code rate and $D(R)$ is the distortion [18]. In the following we discuss the distortion-rate characteristics of the considered two error control schemes.
In the case of MD-FEC the first and the subsequent $v-1$ (redundant) descriptions are encoded independent from each other, and thus, if any of them is received, its distortion is given by (1). We denote the rate allocated to the primary encoding with $R_{1}^{M D F}$ and the rate allocated to the $k^{\text {th }}$ (redundant) copy with $R_{k}^{M D F}$. The redundancy ratio introduced by the MD-FEC is then $\beta=\sum_{k=2}^{v} R_{k}^{M D F} / R_{1}^{M D F}$, and the total rate of the source is $R^{M D F}=R_{1}^{M D F}(1+\beta)$. In the case, when both the primary and some redundant encodings are received, the redundant encodings can not be used to reduce the distortion of the original encoding.

In the case of MDC we denote the total source rate with $R^{M D C}$ and consider the balanced two channel case. We denote the rate allocated to individual descriptions with $R_{1}^{M D C}=R^{M D C} / 2$. The distortion when both descriptions are received, called the central distortion, is denoted by $D_{0}^{M D C}$ and the distortion if only one of the descriptions is received, called the side distortion, is denoted with $D_{1}^{M D C}$. The distortion rate bounds for the 2-channel MDC are [24]

$$
\begin{aligned}
D_{1}^{M D C} & \geq 2^{-2 R^{M D C} / 2} \\
D_{0}^{M D C}\left(D_{1}^{M D C}\right) & \geq 2^{-2 R^{M D C}} \gamma\left(R^{M D C}, D_{1}^{M D C}\right),
\end{aligned}
$$

where $\gamma\left(R^{M D C}, D_{1}^{M D C}\right)=1$ if $2 D_{1}^{M D C}>1+D_{0}^{M D C}$ and

$$
\gamma\left(R^{M D C}, \quad \frac{\left.D_{1}^{M D C}\right)=}{1-\left\{\left(1-D_{1}^{M D C}\right)-\sqrt{\left.\left(D_{1}^{M D C}\right)^{2}-2^{-2 R^{M D C}}\right\}^{2}}\right.}\right.
$$

otherwise. Equation (3) shows that if the side distortion is small then the central distortion is higher than the distortion rate minimum for rate $R(1)$. For a primer on rate distortion theory and multiple description coding see [18].

In general the source rate $R$ (measured in bits per symbol) and the bitrate of the stream $\mathrm{C}$ (measured in bits per second) are related to each other through the symbol rate $\mathrm{S}$ (measured in symbols per second) as $C=S \times R$. For example a video sequence with a small image size can reach a higher source rate $R$ at the same bitrate as another sequence with a big image size. For this reason in the following analysis we will distinguish between these two parameters.

\section{AVERAGE DISTORTION IN A LOSSY ENVIRONMENT}

It is known that losses in the network do not occur independently [25]. The correlation between the loss of subsequent packets can be described for example by the conditional loss probability, the probability that, given that an arbitrary packet is lost the $n^{\text {th }}$ next packet is lost as well. We will denote this probability by $p_{\omega \mid \omega}(n)$. We define $p_{\alpha \mid \alpha}(n)$ as the probability that, given that a packet is not lost the $n^{\text {th }}$ next packet is not lost either. In a similar way we can define $P_{\alpha \mid \omega}(n)$ and $P_{\omega \mid \alpha}(n)$. Based on the average loss probability $P_{\omega}$ and the conditional loss probability one can calculate the probability that two packets $n$ packets apart will be lost simultaneously as $P_{\omega \omega}(n)=P_{\omega} P_{\omega \mid \omega}(n)$. 
Let us consider now a source that has an available rate $R_{a}$ and due to the low delay bounds and low bitrate can introduce either MD-FEC or MDC. For simplicity, we will consider the case of $v=2$. Using the notations introduced in Section 2 the mean distortion bound of the MD-FEC scheme can be calculated as the weighted sum of the distortions of the cases when both descriptions are received, when only one of them is received or when none of them is received

$$
\begin{aligned}
D^{M D F}(\beta)= & \left(p_{\alpha \alpha}(n)+p_{\alpha \omega}(n)\right) D\left(R_{1}^{M D F}\right) \\
& +p_{\omega \alpha}(n) D\left(R_{2}^{M D F}\right)+p_{\omega \omega}(n),
\end{aligned}
$$

where the loss probabilities were defined earlier. For a redundancy ratio $\beta$ the rates of the individual descriptions are $R_{1}^{M D F}=R_{a} /(1+\beta)$ and $R_{2}^{M D F}=R_{a} \beta /(1+\beta)$. The level of redundancy that minimizes (4) is the solution $\beta_{*}^{M D F}$ of

$$
\frac{\partial D^{M D F}(\beta)}{\partial \beta}=0 .
$$

Using the distortion rate function introduced in Section 2 the solution of (5) is

$$
\begin{aligned}
\beta_{*}^{M D F} & =\frac{\ln \left(2^{2 R_{a}}\right)-\ln \left(\frac{p_{\alpha \alpha}(n)+p_{\omega \alpha}(n)}{p_{\omega \alpha}(n)}\right)}{\ln \left(2^{2 R_{a}}\right)+\ln \left(\frac{p_{\alpha \alpha}(n)+p_{\omega \alpha}(n)}{p_{\omega \alpha}(n)}\right)} \\
& =\frac{\ln \left(2^{2 R_{a}}\right)-\ln \left(\frac{1-p_{\omega}(n)}{p_{\omega}(n)\left(1-p_{\omega \mid \omega}(n)\right)}\right)}{\ln \left(2^{2 R_{a}}\right)+\ln \left(\frac{1-p_{\omega}(n)}{p_{\omega}(n)\left(1-p_{\omega \mid \omega}(n)\right)}\right)} .
\end{aligned}
$$

We denote the minimal mean distortion with $D_{*}^{M D F}$. Based on (6) the optimal ratio of redundancy $\beta_{*}^{M D F}$ is positive only if

$$
p_{\omega}>\frac{1}{1+D\left(R_{a}\right)^{-1}\left(1-p_{\omega \mid \omega}(n)\right)}
$$

Thus for a given rate it is not beneficial to use MD-FEC below a certain average loss probability. Furthermore, if $p_{\omega \mid \omega}(n)$ is not close to 1 , the minimum value of $p_{\omega}$ is dominated by $D\left(R_{a}\right)^{-1}$. In general, for any distortion rate function of the form $D(R)=a b^{-c R}$ the limit given in (7) is $p_{\omega}>\frac{1}{1+a D\left(R_{a}\right)^{-1}\left(1-p_{\omega \mid \omega}(n)\right)}$.

By substituting $\beta_{*}^{M D F}$ given by (6) into (4) it can be shown analytically that increasing $n$ decreases the average distortion whenever $p_{\omega}$ satisfies (7) as $p_{\omega \mid \omega}(n)$ is a decreasing function of $n$. This result is in accordance with the empirical observations presented in [12]. If, however, $p_{\omega} \leq 1 /\left(1+D\left(R_{a}\right)^{-1}\right)$ then increasing $n$ does not decrease the distortion. The value $\beta_{*}^{M D F}$ that minimizes the average distortion at a given average loss probability and conditional loss probability is shown in Fig. 1 and 2 for distortions $D\left(R_{a}\right)=10^{-2}$ and $D\left(R_{a}\right)=10^{-4}$ respectively. Comparing the figures shows that the rate of the source and the average loss probability have a big influence on whether or not MD-FEC should be used.

Now we consider the case of the balanced MDC scheme with two descriptions. The total available rate is $R_{a}$, and thus the rates of the individual descriptions are $R_{1}^{M D C}=$

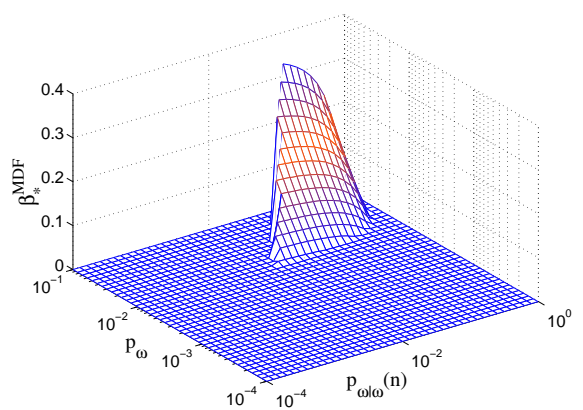

Figure 1: Optimal rate of redundancy vs. average loss probability and conditional loss probability for $D(R)=10^{-2}$.

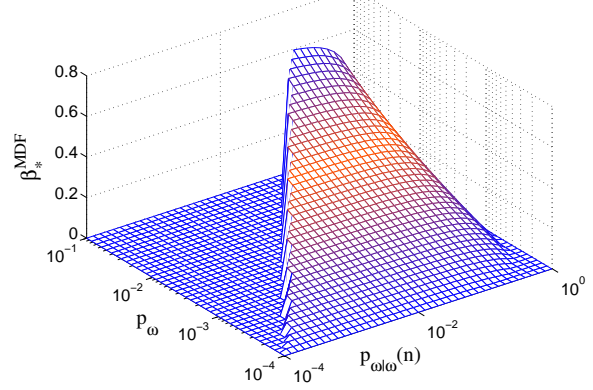

Figure 2: Optimal rate of redundancy vs. average loss probability and conditional loss probability for $D(R)=10^{-4}$.

$R_{2}^{M D C}=R_{a} / 2$. The mean distortion bound can be calculated as

$$
\begin{aligned}
D^{M D C}\left(D_{1}^{M D C}\right)= & p_{\alpha \alpha}(n) D_{0}^{M D C}\left(D_{1}^{M D C}\right) \\
& +\left(p_{\alpha \omega}(n)+p_{\omega \alpha}(n)\right) D_{1}^{M D C}+p_{\omega \omega}(n) .
\end{aligned}
$$

The optimal combination of central and side distortion can be found by setting the first derivative with regard to $D_{1}^{M D C}$ of (8) to zero [20]. The solution is given by

$$
\begin{aligned}
\frac{\partial D_{0}^{M D C}\left(D_{1}^{M D C}\right)}{\partial D_{1}^{M D C}} & =-\frac{p_{\alpha \omega}(n)+p_{\omega \alpha}(n)}{p_{\alpha \alpha}(n)} \\
& =-\frac{p_{\omega}\left(1-p_{\omega \mid \omega}(n)\right)}{1+p_{\omega \mid \omega}(n) p_{\omega}-2 p_{\omega}} .
\end{aligned}
$$

We denote the minimal mean distortion by $D_{*}^{M D C}$. To compare the performance of MD-FEC and MDC we define $\delta_{M D F}^{M D C}=D_{*}^{M D C} / D_{*}^{M D F}$, the ratio of the mean distortion bounds. Similarly we define $\delta^{M D C}=D_{*}^{M D C} /\left(p_{\alpha} D(R)+\right.$ $\left.p_{\omega}\right)$, the ratio of the mean distortion bound with MDC and without any error control. Figs. 3 and 4 show $\delta^{M D C}$ as a function of the average loss probability and the conditional loss probability for distortions $D\left(R_{a}\right)=10^{-2}$ and $D\left(R_{a}\right)=10^{-4}$. They show that the source rate, the average loss probability and the conditional loss probability together determine the potential of MDC to decrease the mean distortion. Figs. 5 and 6 show $\delta_{M D F}^{M D C}$ as a function of the average loss probability and the conditional loss probability for distortions $D\left(R_{a}\right)=10^{-2}$ and $D\left(R_{a}\right)=10^{-4}$ They show that MDC reduces the mean distortion more 
efficient than MD-FEC under all circumstances. The difference, however, is not large, up to a factor of 25 percent. Comparing these figures to Figs. 1 and 2 we see that MDC outperforms MD-FEC primarily in those regions of average loss and conditional loss probability where $\beta_{*}^{M D F}>0$. It follows that error correction with MDC becomes practically useless below the loss probability given in (7).

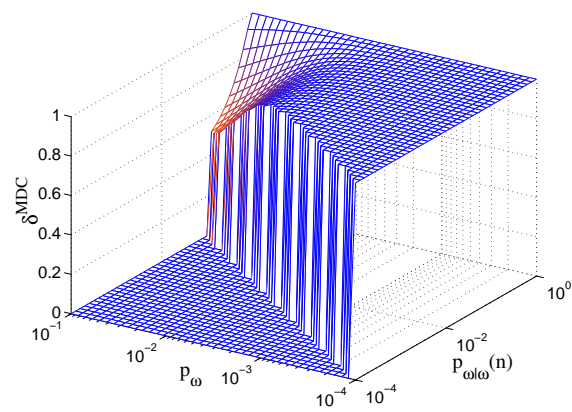

Figure 3: MDC gain vs. average loss probability and conditional loss probability for $D\left(R_{a}\right)=10^{-2}$.

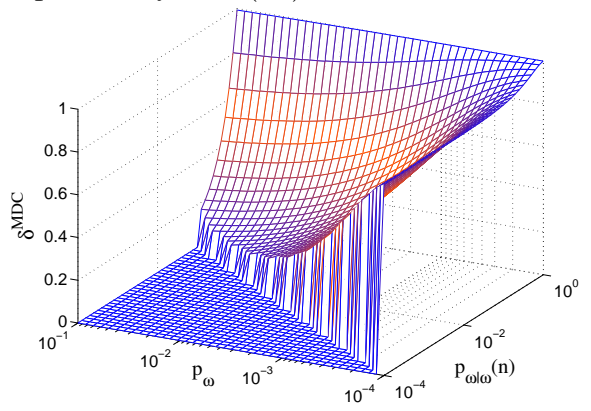

Figure 4: MDC gain vs. average loss probability and conditional loss probability for $D\left(R_{a}\right)=10^{-4}$.

\section{EVALUATION BASED ON MEASURED TRACES}

The value of the parameters $p_{\omega}$ and $p_{\omega \mid \omega}(n)$ used in the evaluation presented above can be taken from measured traces, simulations or mathematical models. In the remainder of the section we use traces of approximately 23000 VoIP calls measured previously between hosts at nine academic sites [26] to calculate these probabilities. Calls were made in a full-mesh on an hourly basis over a period of 15 weeks using a sequence of pre-recorded speech samples at $64 \mathrm{kbps}$, and the logs of the arrival processes were recorded at the receiver sides. For more details on the measurement and a statistical analysis of the measured data see [26]. Since in the absence of losses the optimal rate of redundancy is $\beta_{*}^{M D F}=0$, for the evaluation we selected the traces that experienced losses, and calculated the probabilities $p_{\omega}$ and $p_{\omega \mid \omega}(n)$ for each of the remaining 5700 traces for $n=1,2,4,9$ and a delay jitter buffer of $20 \mathrm{~ms}$. A packet was considered lost if it arrived after the delay limit or it has never arrived. Note that while the first description has a delay jitter limit of $(n+1) 20 \mathrm{~ms}$, the second

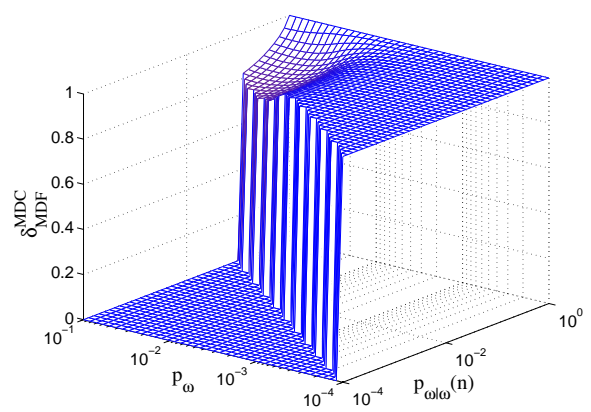

Figure 5: MDC/MD-FEC gain vs. average loss probability and conditional loss probability for $D\left(R_{a}\right)=10^{-2}$.

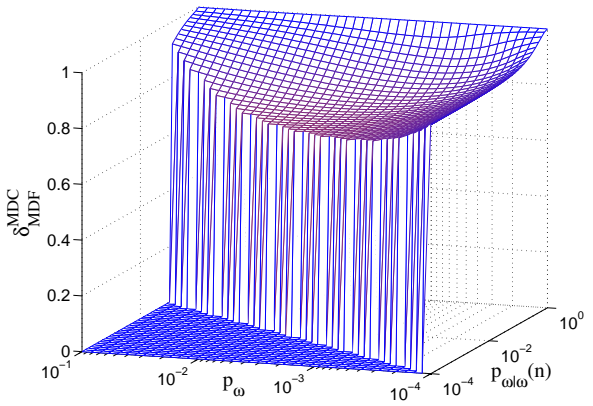

Figure 6: MDC/MD-FEC gain vs. average loss probability and conditional loss probability for $D\left(R_{a}\right)=10^{-4}$.

description has only $20 \mathrm{~ms}$. Using these parameters the delay introduced is $40,60,100$ and $200 \mathrm{~ms}$ given that the packet interarrival time is $20 \mathrm{~ms}$. Based on the probabilities $p_{\omega}$ and $p_{\omega \mid \omega}(n)$ we calculated the optimal MD-FEC and MDC parameters for $D\left(R_{a}\right)=10^{-2}, D\left(R_{a}\right)=10^{-4}$ and $D\left(R_{a}\right)=10^{-6}$ using eqs. (6) and (9).

Figs. 7-15 show the distribution of $\beta_{*}^{M D F}, \delta^{M D C}$ and $\delta_{M D F}^{M D C}$ for $D\left(R_{a}\right)=10^{-2}, D\left(R_{a}\right)=10^{-4}$ and $D\left(R_{a}\right)=$ $10^{-6}$. For example, Fig. 7 shows that for an available code rate $R_{a}=-\log _{2} \sqrt{0.01}$ in the case of 90 percent of the calls that experienced any losses the optimal rate of redundancy $\beta_{*}^{M D F}$ is less than 10 percent. The figures show that for $D\left(R_{a}\right)=10^{-2}$ MD-FEC should not be used in more than 85 percent of the calls that experience losses, as $p_{\omega}(n)$ and $p_{\omega \mid \omega}(n)$ do not satisfy inequality (7). Furthermore the gain of using MDC is less than 10 percent for $90-95$ percent of the calls, depending on the value of $n$. At the same time, the gain of MDC over MD-FEC is less than 10 percent for about 95 percent of the calls. Thus for low code rates both MD-FEC and MDC are practically useless in today's Internet. For $D\left(R_{a}\right)=10^{-4}$ both MD-FEC and MDC are more efficient. MDC reduces the average distortion by at least 50 percent for up to 30 percent of the calls, and outperforms MD-FEC by more than 10 percent for up to 40 percent of the calls depending on the value of $n$. For $D\left(R_{a}\right)=10^{-6}$, MDC reduces the average distortion by at least 20 percent for up to 50 percent of the calls, and by at least 90 percent for up to 30 percent of the calls. Furthermore MDC outperforms MD-FEC by at least 25 percent 


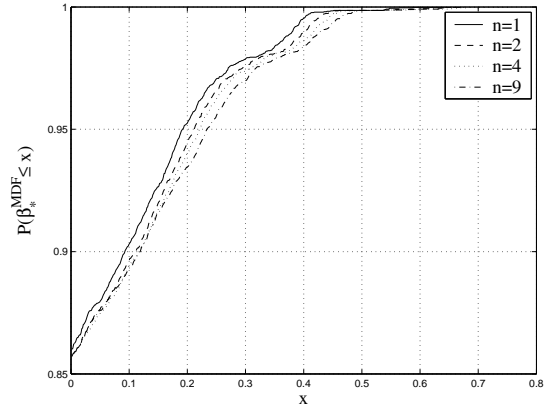

Figure 7: Distribution of the optimal value of $\beta_{*}^{M D F} . D\left(R_{a}\right)=10^{-2}$.

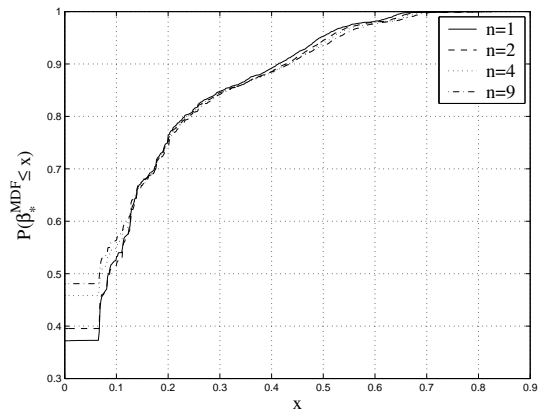

Figure 10: Distribution of the optimal value of $\beta_{*}^{M D F} . D\left(R_{a}\right)=10^{-4}$.
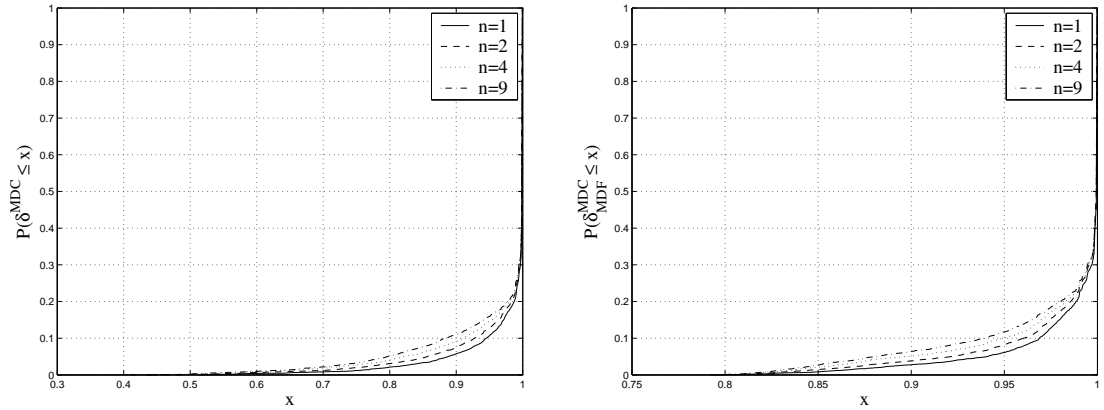

Figure 8: Distribution of the MDC gain. $D\left(R_{a}\right)=10^{-2}$.

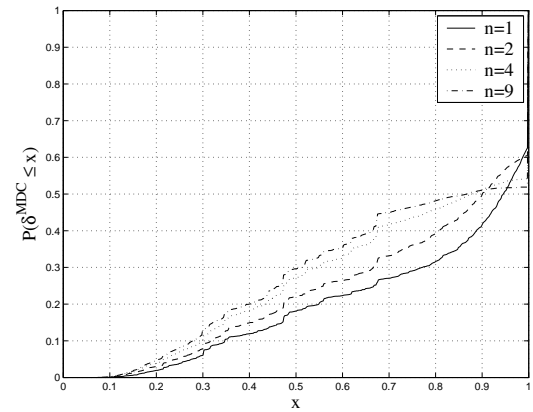

Figure 11: Distribution of the MDC gain. $D\left(R_{a}\right)=10^{-4}$.
Figure 9: Distribution of the MDC/MD-FEC gain. $D\left(R_{a}\right)=10^{-2}$.

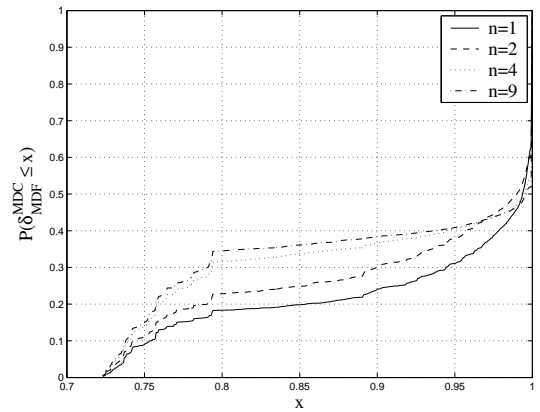

Figure 12: Distribution of the MDC/MD-FEC gain. $D\left(R_{a}\right)=10^{-4}$. for up to 30 percent of the calls. Based on these results we conclude that for high code rates MDC can decrease the average distortion considerably better than MD-FEC, and thus its use can be feasible despite of its complexity compared to MD-FEC. The figures also confirm the observation in [12] that in general for higher values of $n$ both MD-FEC and MDC perform better as losses occur less correlated. While the difference between the results is not significant for $D\left(R_{a}\right)=10^{-2}$, it reaches up to 20 percent for higher available rates.

\section{CONCLUSIONS}

In this paper we presented an analytical evaluation of the potential of forward error correction and multiple description coding to recover from losses. We concluded that MDC gives better performance in all circumstances. The comparison is based on the loss probabilities, thus it is independent of the traffic parameters. We showed that for a big class of distortion rate models the optimal ratio of redundancy is mainly determined by the average packet loss probability. Using traces of measurements performed on the Internet we concluded that for low code rates neither MD-FEC nor MDC can achieve significant improvements, while for high code rate streams MDC outperforms MD-FEC significantly. Based on the results presented in this paper we believe that multiple description coding is an appealing error control solution for delay sensitive traffic with a high code rate in an environment with correlated packet losses like the Internet.

\section{REFERENCES}

[1] R. Ramjee, J. Kurose, D. Towsley, and H. Schulzrinne, "Adaptive playout mechanisms for packetized audio applications in wide-area networks," in Proc. of IEEE INFOCOM, 1994.

[2] J. Rosenberg, L. Qiu, and H. Schulzrinne, "Integrating packet FEC into adaptive voice playout buffer algorithms on the Internet," in Proc. of IEEE INFOCOM, pp. 1705$1714,2000$.

[3] C. Perkins, O. Hodson, and V. Hardman, "A survey of packet loss recovery for streaming audio," IEEE Network, 1998.

[4] Y. Wang and Q. Zhu, "Error control and concealment for video communication: a review," Proc. IEEE, vol. 86, no. 5, pp. 974-997, 1998.

[5] J. C. Bolot, S. Fosse-Parisis, and D. Towsley, "Adaptive FEC-based error control for Internet telephony," in Proc. of IEEE INFOCOM, pp. 1453-1460, 1999.

[6] V. A. Vaishampayan, "Design of multiple description scalar quantizers," IEEE Trans. Inform. Theory, vol. 39, pp. 821834, May 1993.

[7] E. Biersack, "Performance evaluation of forward error correction in ATM networks," in Proc. of ACM SIGCOMM, pp. 248-257, August 1992. 


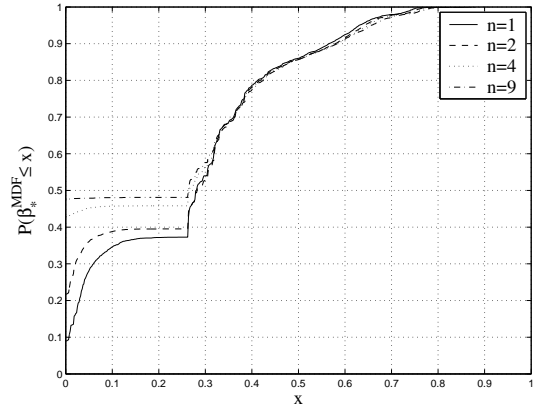

Figure 13: Distribution of the optimal value of $\beta_{*}^{M D F} . D\left(R_{a}\right)=10^{-6}$.

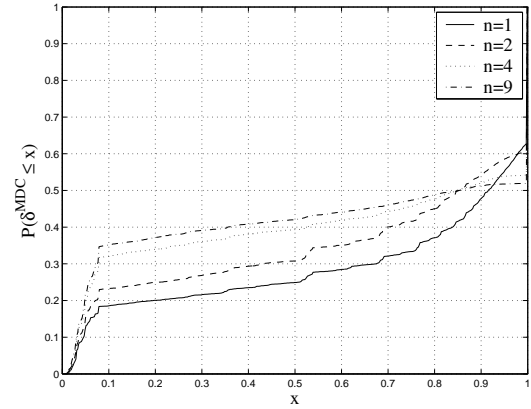

Figure 14: Distribution of the MDC gain. $D\left(R_{a}\right)=10^{-6}$.

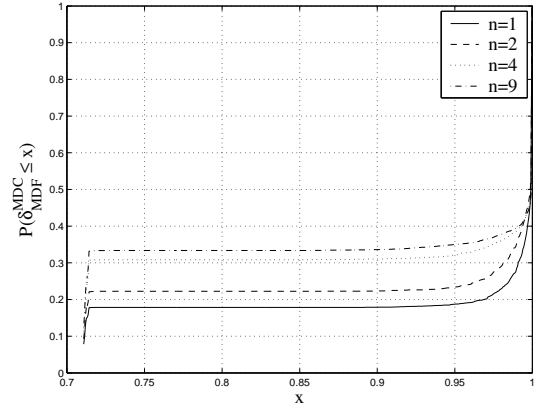

Figure 15: Distribution of the MDC/MD-FEC gain. $D\left(R_{a}\right)=10^{-6}$.
[8] K. Kawahara, K. Kumazoe, T. Takine, and Y. Oie, "Forward error correction in ATM networks: An analysis of cell loss distribution in a block," in Proc. of IEEE INFOCOM, pp. 1150-1159, June 1994.

[9] G. Dán, V. Fodor, and G. Karlsson, "On the effects of the packet size distribution on FEC performance," Computer Networks, to appear, 2005.

[10] The Multimedia Integrated Conferencing for European Researchers (MICE) Project, "RAT: Robust Audio Tool." http://www-mice.cs.ucl.ac.uk/multimedia/software/rat.

[11] A. V. García and S. Fosse-Parisis, "Freephone audio tool." http://www-sop.inria.fr/rodeo/fphone.

[12] I. Kouvelas, O. Hodson, W. Hardman, and J. Crowcroft, "Redundancy control in real-time Internet audio conferencing," in Proc. of International Workshop on Audio-Visual Services Over Packet Networks, September 1997.

[13] M. Podolsky, C. Romer, and S. McCanne, "Simulation of FEC-based error control for packet audio on the Internet," in Proc. of IEEE INFOCOM, pp. 505-515, 1998.

[14] E. Altman, C. Barakat, and V. M. Ramos, "Queueing analysis of simple FEC schemes for IP telephony," in Proc. of IEEE INFOCOM, pp. 796-804, 2001

[15] E. Altman, C. Barakat, and V. Ramos, "On the utility of FEC mechanisms for audio applications," in Proc. of Quality of Future Internet Services, LNCS 2156, pp. 45-56, 2001.

[16] C. E. Shannon, "A mathematical theory of communication," Bell Syst. Tech. J., pp. 379-423, 1948.

[17] S. Vembu, S. Verdu, and Y. Steinberg, "The source-channel separation theorem revisited," IEEE Trans. Inform. Theory, vol. 41, pp. 44-54, January 1995.

[18] V. K. Goyal, "Multiple description coding: Compression meets the network," IEEE Signal Processing Mag., Sept. 2001.

[19] J. Apostolopoulos, T. Wong, W. Tan, and S. Wee, "On multiple description streaming with content delivery networks," in Proc. of IEEE INFOCOM, pp. 1736-1745, 2002.

[20] M. Y. Kim and B. W. Kleijn, "Rate-distortion comparisons between FEC and MDC based on Gilbert channel model," in Proc. of IEEE ICON, pp. 495-500, 2003.

[21] P. Hedelin and J. Skoglund, "Vector quantization based on gaussian mixture models," IEEE Trans. Speech Audio Processing, vol. 8, no. 4, pp. 385-401, 2000.
[22] A. D. Subramaniam, W. Gardner, and B. Rao, "Joint source-channel decoding of speech spectrum parameters over erasure channels using gaussian mixture models," in Proc. of IEEE Conference on Acoustics, Speech, and Signal Processing, vol. 1, pp. 120-129, April 2003.

[23] R. Zamir, "Gaussian codes and Shannon bounds for multiple descriptions," IEEE Trans. Inform. Theory, vol. 45, pp. 2629-2635, November 1999.

[24] L. Ozarow, "On a source-coding problem with two channels and three receivers," Bell Syst. Tech. Journal, vol. 59, pp. 1909-1921, December 1980.

[25] I. Cidon, A. Khamisy, and M. Sidi, "Analysis of packet loss processes in high speed networks," IEEE Trans. Inform. Theory, vol. 39, no. 1, pp. 98-108, 1993.

[26] I. Marsh, F. Li, and G. Karlsson, "Wide area measurements of VoIP quality," in Proc. of Quality of Future Internet Services, pp. 63-72, October 2003. 